\title{
ESPONDILITE ANQUILOSANTE: O EXERCÍCIO FÍSICO COMO REABILITAÇÃO E PROMOTOR DA QUALIDADE DE VIDA
}

\author{
ANKYLOSING SPONDYLITIS: THE PHYSICAL EXERCISE \\ AS A FORM OF REHABILITATION AND PROMOTER OF QUALITY OF LIFE
}

\author{
AUTOR \\ Rosa Maria Dantas Costa \\ Maria Dolores Gonzalez Monteagudo \\ ${ }^{1}$ Universidade Fernando Pessoa
}

ESPONDILITE ANQUILOSANTE: O EXERCÍCIO FÍSICO COMO REABILITAÇÃO E PROMOTOR DA QUALIDADE DE VIDA

4(2): 11-20

\section{PALAVRAS-CHAVE}

espondilite anquilosante; exercício físico; motricidade humana; qualidade de vida.

\section{KEYWORDS}

ankylosing spondylitis; physical exercise; human kinetics; quality of life.
RESUMO

Introdução: o objectivo principal do estudo foi verificar a relação entre a prática de exercício físico e a qualidade de vida em doentes com Espondilite Anquilosante.

Material e métodos: procedeu-se a um estudo quantitativo, com abordagem descritiva correlacional. Como instrumento de colheita de dados utilizou-se um questionário, com duas partes, a primeira recolheu informações sócio-demográficas, realização ou não de reabilitação e prática de exercício físico e a segunda, pelo questionário de estado de saúde, SF36.

Para o tratamento de dados recorreu-se ao programa estatístico SPSS (Statistical Package for Social Sciences], versão 12.0. Em termos de técnicas estatísticas, foram utilizadas a estatística descritiva e a inferencial. Resultados: participaram no estudo 19 pacientes com Espondilite Anquilosante, 10 do género feminino e 9 do género masculino, com média de idades de 46,58 anos. Os primeiros sintomas apareceram em média aos 27,53 anos; o tempo decorrido até ser estabelecido o diagnóstico foi de 6,88 anos e apresentavam, em média, 10,83 anos de evolução da doença. Relativamente à prática de exercício físico, comprovou-se que 10 indivíduos praticavam regularmente e os restantes 9 não efectuavam qualquer tipo de exercício físico.

Conclusões: observou-se que a idade, idade do diagnóstico e o tempo de estabelecimento do diagnóstico influenciam positiva e negativamente na qualidade de vida e que subsistem diferenças na percepção do estado de saúde em relação à prática de exercício físico.

\begin{abstract}
Introduction: the main goal of this study was to verify if the practice of physical exercise is related to the quality of life in patients with Ankylosing Spondylitis.
\end{abstract}

Material and methods: it was made a quantitative study, following a correlative and descriptive approach. As a means of data collection it was used a questionnaire that it was organized in two parts, the first one to get information about the social-demographic level, whether if it makes or not rehabilitation and about the practise of exercise and the second one related to the quality of life questionnaire, SF36.

For the data treatment, it was used the statistical program SPSS (Statistical Package for Social Sciences], version 12.0. The statistical techniques used were the descriptive and deductive ones.

Results: it involved 19 patients with Ankylosing Spondylitis, 10 females and 9 males, whose average age was 46,58 years old. The first symptoms occurred, in average, at the age of 27,53; the lapse of time between the disease appearance and the diagnosis was of 6,88 years and the patients presented, in average, 10,83 years of evolution of disease. Concerning physical exercise, the study proved that 10 patients practised it regularly, while the other 9 didn't practise any type of physical exercise.

Conclusions: It was possible to conclude that the age, age of diagnosis and the lapse of time between the disease appearance and the diagnosis influence positive and negative in quality of life and that there are different perceptions regarding the relation between health and physical exercise. 


\section{INTRODUC̣ÃO}

A Espondilite Anquilosante (EA) é uma doença reumatológica, com um quadro evolutivo bastante marcado e com um comprometimento progressivo axial ascendente com consequências nefastas para o doente ${ }^{19}$. Contudo, autores ${ }^{21,25}$ referem que a prática de exercício físico, de forma correcta e regular, é de importância vital para poder dispor de bons resultados a longo prazo, uma vez que estes ajudam a melhorar a postura corporal, a amplitude dos movimentos torácicos, a mobilidade da coluna vertebral, a qualidade de vida e o estado de saúde. Outros investigadores, asseveram que, o exercício físico também previne ou adia e minimiza as deformidades inerentes à doença ${ }^{28,22}$.

Segundo alguns autores, é actualmente reconhecida a importância de abordagens não farmacológicas na intervenção de diversas condições do foro reumático como principal medida terapêutica tendo os fármacos um papel adjuvante. A fisioterapia e o desenvolvimento de programas de exercício constituem uma parte fundamental no tratamento destas patologias.

Inicia-se este ponto com uma questão prévia: actividade física ou exercício físico? Vale a pena salientar que actividade física "(...) reporta-se a qualquer movimento corporal produzido pelos músculos esqueléticos, com consequente gasto de energia", enquanto que exercício físico "(...) compreende actividade física planeada, estruturada e repetitiva da qual resulta a manutenção ou melhoria de uma ou mais facetas da condição física e da saúde"s. Neste estudo, a terminologia utilizada foi o exercício físico, uma vez que o seu objectivo foi avaliar a influência daquele na qualidade de vida dos pacientes.

0 exercício físico nas patologias reumáticas tem como desígnio trabalhar força muscular, amplitude articular, propriocepção, a postura corporal e consequentemente meIhorar performances funcionais.

Na EA, o exercício físico permite restituir ao sistema osteoarticular os arcos de amplitude de movimento que a inflamação fez perder; proporcionar aos centros cefálicos do esquema corporal um quantum de aferências proprioceptivas provenientes dos mecanismos capsulo-ligamentares das articulações interapofisárias e da musculatura satélite loco-regional; dar à musculatura satélite das articulações bloqueadas em rigidez reversivel a possibilidade de retomarem o tonus normal, após contracções por estímulos nervosos centrais e relaxamento/ /alongamento por reciprocidade da contracção; proporciona ao tecido fibroblástico em evolução cicatricial um estímulo para metaplasia em fibrocartilagem, permitindo a conservação de amplitude de movimento com relevância funcional; ministrar estímulos vectoriais para manutenção da massa óssea e da normal arquitectura trabecular, limitando e revertendo a remodelação óssea em padrões disfuncionais ${ }^{24,33}$.

0 exercício físico pode contribuir para o controlo da mensagem nociceptiva, quer promovendo o seu bloqueio medular ("gate control"), através da estimulação das fibras mielinizadas de grande calibre, priorizando a via rápida, quer através da activação do sistema opióide endógeno, com libertação de substâncias "morfina-like", o que limita a actividade de neurotransmissores envolvidos nas mensagens nociceptivas a nível medular. Outro aspecto abordado acerca dos benefícios do exercício físico, é o facto deste proporcionar uma estimulação proprioceptiva, factor de estabilidade dinâmica, promovendo uma modulação e coordenação da actividade muscular. Este tipo de estimulação tem como base múltiplas mensagens aferentes, provenientes de vários receptores periféricos (Ruffini, $\mathrm{Pa}$ cini, Golgi e fuso neuromuscular], que sofrem processos complexos de integração, quer a nível medular (excitatório la, inibição recíproca la e inibição lb), quer a nível supra-segmentar ${ }^{20}$.

A qualidade de vida em 1986, era definida pela OMS como a percepção dos indivíduos ou grupos de que as suas necessidades estão a ser satisfeitas e de que não thes estão a ser negadas oportunidades de alcançar a felicidade e satisfação completas.

Numa pessoa com EA, a qualidade de vida, está baseada na percepção da malha de consequências da doença nos seguintes aspectos: sintomas, aptidão física, capacidade de trabalho, funções e interacções psicossociais, efeitos colaterais indesejáveis da medicação ${ }^{23}$ e também dos custos biológicos e financeiros, directos e indirectos ${ }^{21}$.

Contudo, a diminuição das capacidades funcionais, $\mathrm{o}$ aumento da dor e as alterações psicológicas, resultantes da doença, são os factores que mais contribuem para a deterioração da qualidade de vida nestes doentes ${ }^{4}$.

A promoção da qualidade de vida implica a adopção de um estilo de vida adequado à sua doença e que seja promotor da saúde ${ }^{23}$. Investigações realizadas demonstraram que o exercício físico contribui, entre outros benefícios, para a diminuição da dor, aumento do bem-estar e, consequentemente, melhor qualidade de vida ${ }^{18,29,31}$. 
Independentemente da promoção de uma política de saúde que engloba naturalmente todas as formas de cuidados médicos e farmacêuticos, incluindo reabilitação médica e funcional, é importante a prestação de serviços por técnicos especializados, numa perspectiva de melhoria da qualidade de vida das pessoas com Espondilite Anquilosante. 0 primeiro autor a abordar este tema ${ }^{26}$, asseverou que: "[...] o desporto, a dança, a ergonomia, a reabilitação física e psicomotora e enfim os vários aspectos da Motricidade Humana, do jogo ao trabalho, passando pela saúde, - lazer e a educação, são as especialidades que despontam da Ciência da Motricidade Humana".

É da competência destes profissionais a implementação de projectos de intervenção nas várias áreas atrás referidas, com o intuito de melhorar toda a qualidade de vida destes doentes. No entanto, toda a intervenção deve ser planeada e estruturada, observando o ser humano na integridade das suas funções e das suas potencialidades, numa perspectiva holística. Não se pretende exclusivamente realizar a reabilitação propriamente física, mas também operar ao nível dos aspectos psico-socio-culturais. Pretendeu-se com esta investigação, verificar se estes doentes praticam exercício físico, com que finalidade o fazem, identificar quais os benefícios que os praticantes adquirem aquando da prática do exercício físico, assim como conhecer que repercussões têm ao nível da qualidade de vida em relação com a saúde e verificar se algumas variáveis, como a idade, idade do diagnóstico e o tempo de estabelecimento do diagnóstico influenciam a qualidade de vida em doentes com EA.

\section{METODOLOGIA}

\section{Amostra}

Os pacientes com Espondilite Anquilosante inscritos no Núcleo Regional de Ponte de Lima da Associação Nacional de Espondilite Anquilosante, que representam vinte e um pacientes. A amostra foi constituída por dezanove pacientes que representam o total de indivíduos que aceitaram participar no estudo. Os critérios de selecção da amostra foram: os pacientes tinham que ser associados do núcleo, doentes com Espondilite Anquilosante, com capacidade físicas e psíquicas para responder ao questionário e com mais de 14 anos de idade lum dos requisitos para a aplicação do questionário - SF36].

Para que todos os princípios éticos inerentes à investigação fossem cumpridos, procedeu-se à explicação do estudo à presidente do núcleo e aos pacientes, a fim de receber a respectiva autorização. Foi elaborado um consentimento informado, que consistia num formulário, onde os sujeitos declaravam que foram bem informados sobre a investigação e que aceitavam participar nela.

\section{Variáveis estudadas}

A primeira parte do questionário, além das questões que caracterizavam a amostra em termos sócio-demográficos, permitia também recolher informação dos pacientes quanto à realização de reabilitação, à prática de exercício físico, modalidade e o motivo que os leva a participar. Informações relativas à qualidade de vida recolheram-se na segunda parte do questionário de estado de saúde [SF-36], para o qual foi efectuado pedido de autorização ao autor e obtida respectiva autorização. Este é considerado um instrumento de medida genérico de saúde, que se destina a medir a percepção do estado de saúde e da qualidade de vida, que representam valores humanos básicos relevantes à funcionalidade e ao bem-estar de cada um. 0 formato final contém 36 itens cobrindo oito dimensões do estado de saúde e que detecta tanto os aspectos positivos da saúde, como os negativos da saúde ${ }^{14}$. Permite medir oito principais dimensões em saúde, a função física (FF) destina-se a medir o impacto das limitacões físicas limportantes para o desempenho das actividades da vida diária] na qualidade de vida, sejam elas em situações como tomar banho ou vestir-se sozinho/a, praticar desportos mais exigentes fisicamente ou mesmo carregar os sacos das compras, ajoelhar-se ou andar uma determinada distância. As escalas de desempenho medem o impacto das limitações em saúde devidas a problemas físicos (DF) ou a problemas emocionais [DE], ao tipo e quantidade de trabalho realizado, à necessidade de reduzir o trabalho ou à dificuldade de o realizar. A escala para a dor (DC) representa a intensidade e o desconforto que ela causa e o modo como ela interfere com o trabalho normal. A componente saúde em geral (SG) mede a percepção holística da saúde, englobando a saúde actual, a resistência à doença e o aspecto saudável e a vitalidade (VT) inclui os niveis de energia e de fadiga. A dimensão função social (FS) capta a quantidade e a qualidade das actividades sociais, o impacto dos problemas físicos e emocionais nestas actividades e a saúde mental (SM) inclui os conceitos de ansiedade, de depressão, de perda de controlo comportamental ou emocional e de bem-estar psicológico ${ }^{12,13}$. 


\begin{tabular}{lcc}
\hline & $\begin{array}{c}\text { Número } \\
\text { de casos ( } \mathbf{n}=19)\end{array}$ & Percentagem \\
\hline Género & & \\
Masculino & 9 & $47,5 \%$ \\
Feminino & 10 & $52,6 \%$ \\
Estado civil & & \\
Solteiro & 4 & $21,1 \%$ \\
Casado & 14 & $73,7 \%$ \\
Viúvo & 1 & $5,3 \%$ \\
Actividade profissional & & \\
Reformada & 1 & $5,3 \%$ \\
Doméstica & 4 & $21,1 \%$ \\
Comerciante & 1 & $5,3 \%$ \\
Manobrador de máquinas & $5,3 \%$ \\
Oficial de justiça & 1 & $5,3 \%$ \\
Contabilista & 1 & $5,3 \%$ \\
Carpinteiro & 1 & $5,3 \%$ \\
Arquitecto & 1 & $5,3 \%$ \\
Escriturário & 1 & $5,3 \%$ \\
Eng. Civil & 1 & $5,3 \%$ \\
Emp. Fabril & 1 & $5,3 \%$ \\
Mecânico & 1 & $5,3 \%$ \\
Costureira & 1 & $5,3 \%$ \\
Bibliotecária & 1 & $5,3 \%$ \\
Aux. acção educativa & 1 & $5,3 \%$ \\
Professor & 1 & $5,3 \%$ \\
\hline
\end{tabular}

\section{QUADRO1}

Características sócio-demográficas da amostra.
As variáveis estranhas identificadas no presente estudo reportam-se ao nível de desenvolvimento da doença e nível de funcionalidade dos pacientes.

\section{Tratamento estatístico}

Para o tratamento estatístico dos dados utilizou-se o programa estatístico SPSS (Statistical Package for Social Sciences], versão 12.0, e

\begin{tabular}{lcccc} 
Variável & Média & Min. & Máx. & D/P \\
\hline Idade & 46,58 & 24 & 86 & 15,94 \\
Idade dos primeiros sintomas & 27,53 & 15 & 50 & 8,60 \\
Idade do diagnóstico & 35,00 & 19 & 69 & 12,63 \\
Tempo até o diagnóstico & 6,88 & 0 & 39 & 11,03 \\
Duração da doença & 10,83 & 0 & 35 & 9,45 \\
\hline
\end{tabular}

QUADRO2

Características sócio-demográficas da amostra. as técnicas estatísticas utilizadas foram a descritiva e a inferencial. $\mathrm{Na}$ análise descritiva os resultados expressaram-se com médias, mínimo, máximo e desvio padrão. Recorreu-se o teste de Mann-Whitney para verificar a diferença de médias e a correlação $\mathbf{R}$ de Pearson para verificar associações entre variáveis. Os resultados eram significativos quando $p<0,05$.

RESULTADOS

\section{Estatística descritiva}

Com base no quadro 1, pode-se aferir que a amostra era constituída por 10 elementos do género feminino [52,6\%] e 9 do género masculino $[47,4 \%$ ). Quanto ao estado civil, 14 elementos eram casados, 4 solteiros e um viúvo. Profissionalmente apresentavam profissões muito variadas.

A média de idade dos inquiridos (quadro 2) situou-se nos 46,58 anos e a idade de aparecimento dos primeiros sintomas nos 27,53 anos, com uma idade mínima de 15 e máxima de 50 anos. Quanto à idade do diagnóstico, a média estabeleceu-se nos 35 anos, o tempo de estabelecimento do diagnóstico nos 6,88 anos, com um máximo de 39 anos e os pacientes apresentavam uma duração da doença de 10,83 anos.

Quanto à realização de reabilitação, a maioria dos pacientes mencionou afirmativamente. Realizavam periodicamente, com o objectivo de prevenir a evolução da doença, mas, também para tratamento da sintomatologia e a modalidade terapêutica realizada pela maioria dos pacientes era a hidroterapia (quadro 3). Dos inquiridos, 52,6\% atestaram 
praticar exercício físico e $47,4 \%$ referiram não cumpriam qualquer tipo de exercício físico. Das modalidades praticadas, confirmou-se que a natação/hidroginástica representa a modalidade praticada em maior percentagem, a seguir a bicicleta, caminhadas e os exercícios diários. Quanto à frequência semanal $\mathbf{3 0} \%$ realiza uma a duas vezes por semana, igual percentagem cinco a seis vezes, e $20 \%$ realiza três a quatro vezes, igual percentagem aos que praticam diariamente. Os motivos da prática de exercício físico, referidos pelos pacientes foram: com o intuito de se sentirem melhor, por aconselhamento médico, com o objectivo de melhorar o seu estado de saúde, para retardar a evolução da doença e para conviver com amigos.

Relativamente às componentes do SF-36 (quadro IV), a função física [FF] apresentou um valor médio de 50,26 , a componente desempenho físico (DF) de 50,00 e a dor corporal (DC) 56,84 . Na saúde geral (SG) obteve-se um valor médio de 61,58 , na vitalidade (VT) de 50,66 e na função social (FS) de 50,00 . 0 valor médio na componente desempenho emocional (DE) aferido foi de 67,98 e na saúde mental (SM) de 54,74. 0 valor mínimo $(12,50)$ observado reporta-se ao nível do desempenho físico e o máximo (100) ao nível do desempenho emocional.

\section{Estatistica inferencial}

0 quadro 4 comprova que o género feminino apresentou valores médios inferiores ao género masculino nas componentes FF, DF, SG e DE. Contudo, estas diferenças, como se pode verificar não são estatisticamente significativas $(p>0,05)$ em qualquer uma das dimensões.

\begin{tabular}{lcc}
\hline & $\begin{array}{c}\text { Número } \\
\text { de casos (n=19) }\end{array}$ & Percentagem \\
\hline Reabilitação & 7 & $36,8 \%$ \\
Não & 12 & $63,2 \%$ \\
Sim & & \\
Frequência da reabilitação & 8 & $66,7 \%$ \\
Periodicamente & 4 & $33,3 \%$ \\
Ocasionalmente & & \\
Finalidade da reabilitação & 5 & $41,7 \%$ \\
$\quad$ Tratamento dos sintomas & 7 & $58,3 \%$ \\
Prevenção e tratamento & & \\
Tipo de reabilitação & 7 & $58,3 \%$ \\
Hidroterapia & 1 & $8,3 \%$ \\
Ginásio & 2 & $16,7 \%$ \\
Ginásio e hidroterapia & 2 & $16,7 \%$ \\
Não sabe & & \\
\hline
\end{tabular}

QUADRO3

Caracterização da amostra referente à realização de reabilitação.

\begin{tabular}{lcc} 
& $\begin{array}{c}\text { Número } \\
\text { de casos }(\mathbf{n}=19)\end{array}$ & Percentagem \\
\hline Prática de exercício físico & 9 & $47,4 \%$ \\
Não & 10 & $52,6 \%$ \\
Sim & & \\
Modalidade praticada & $7 / 10$ & $70 \%$ \\
Natação/hidroginástica & $5 / 10$ & $50 \%$ \\
Bicicleta & $4 / 10$ & $40 \%$ \\
Caminhadas & $5 / 10$ & $50 \%$ \\
Exercícios diários & $2 / 10$ & $20 \%$ \\
Ginástica & $1 / 10$ & $10 \%$ \\
Outra & & $30 \%$ \\
Frequência semanal & 3 & $20 \%$ \\
1-2 vezes & 2 & $30 \%$ \\
$3-4$ vezes & 3 & $20 \%$ \\
$5-6$ vezes & 2 & $80 \%$ \\
Diariamente & & $30 \%$ \\
Motivos da prática & $3 / 10$ & $80 \%$ \\
Convívio & $8 / 10$ & $60 \%$ \\
Retardar evolução da doença & $4 / 10$ & \\
Melhorar o estado de saúde & $6 / 10$ & \\
Aconselhamento médico & $8 / 10$ & \\
Sentir melhor & & \\
\hline
\end{tabular}

QUADRO4 Caracterização da amostra referente à prática de exercício físico. 


\begin{tabular}{lcccc}
\hline Componentes & Média & Min. & Máx. & D/P \\
\hline Função Física (FF) & 50,26 & 15,00 & 90,00 & 24,24 \\
Desempenho Físico (DF) & 50,00 & 12,50 & 81,25 & 20,94 \\
Dor Corporal (DC) & 56,84 & 30,00 & 90,00 & 20,83 \\
Saúde Geral (SG) & 61,58 & 45,00 & 85,00 & 10,00 \\
Vitalidade (VT) & 50,66 & 25,00 & 62,50 & 9,97 \\
Função Social (FS) & 50,00 & 37,50 & 62,50 & 8,33 \\
Desempenho emocional (DE) & 67,98 & 25,00 & 100,00 & 23,12 \\
Saúde Mental (SM) & 54,74 & 40,00 & 65,00 & 8,74 \\
\hline
\end{tabular}

QUADRO5

Estatísticas descritivas das dimensões do SF36.
Foi possivel verificar (quadro 6), que existe relação entre idade e algumas das oito dimensões da qualidade de vida. Encontrou-se a idade e a dimensão FF, em que quanto maior a idade do paciente, menor é a função física e uma correlação moderada positiva entre a idade e a DC que sugestiona que o aumento da idade contribui para o aumento da dor corporal.

Quando se investigou associações entre o tempo de estabelecimento do diagnóstico (TED) e as oito dicontraram-se correlações significacorrelação negativa moderada entre mensões da qualidade de vida, en- tivas nas componentes FF, DF, FS, DC e VT. As correlações negativas moderadas entre a FF, o DF e o tempo de estabelecimento do diagnóstico, sugerem que o aumento deste contribui negativamente, tanto nas funções, como no desempenho físico. As relações positivas encontradas entre o tempo de estabelecimento do diagnóstico e as componentes FS, DC e VT, permitem aferir que o tempo que demora a ser comprovado o diagnóstico contribui para aumentar a função social, a vitalidade mas também a dor percepcionada pelos pacientes.
Verificou-se que existe relação entre idade do diagnóstico (ID) e as dimensões FF e DF, em que o aumento da idade do diagnóstico diminui a função e do desempenho físico. Constatou-se, igualmente, uma correlação moderada positiva entre a idade do diagnóstico e as componentes DC e VT, que, com o aumento da idade do diagnóstico, a dor aumenta, e maiores são os niveis de energia que os pacientes percepcionam.

Para verificar se existiam diferenças nas componentes da qualidade de vida entre os praticantes e os não praticantes de exercício físico, compararam-se as médias e foi possivel aferir, que os pacientes que praticavam exercício físico apresentavam valores médios superiores na dor, função física, desempenho físico e emocional, na saúde geral e mental comparando com os que não praticam. Estas diferenças, como se pode analisar, são estatisticamente significativas nas componentes função física, desempenho físico, dor corporal, desempenho emocional, saúde mental e vitalidade (quadro 7).

\begin{tabular}{lccc} 
Componentes & † & $0^{n}$ & $\boldsymbol{p}$ \\
\hline Função Física (FF) & 46,00 & 55,00 & ns \\
Desempenho Físico (DF) & 46,88 & 53,47 & ns \\
Dor Corporal (DC) & 65,00 & 47,78 & ns \\
Saúde Geral (SG) & 59,50 & 63,89 & ns \\
Vitalidade (VT) & 51,88 & 49,31 & ns \\
Função Social (FS) & 52,50 & 47,22 & ns \\
Desempenho emocional (DE) & 67,50 & 68,52 & ns \\
Saúde Mental (SM) & 55,50 & 53,89 & ns \\
\hline
\end{tabular}

\section{QUADRO6}

Diferença de médias de acordo com o género.

ns: diferença não significativa; $p$ : nível de significância; ᄋ̣: feminino; ơ: masculino

\section{DISCUSSÃO}

0 presente estudo pretendia avaliar a qualidade de vida em doentes com Espondilite Anquilosante, analisar se estes doentes praticam exercício físico e se este influencia na percepção do estado de saúde dos mesmos, verificar se a prática do exercício físico apresenta benefícios, isto é, se funciona como uma modalidade terapêutica e com repercussões ao nível da qualidade de vida dos pacientes. 
A amostra apresentou uma média de idade dos primeiros sintomas de 27,53 anos vai de encontro à bibliografia, que menciona que a EA se caracteriza como uma patologia com início na segunda década de vida, com início por volta da adolescência e raramente surge após os 45 anos de idade ${ }^{25}$. Todavia, muitas pessoas com EA só vêm a ter conhecimento do diagnóstico da sua doença em idade avançada, talvez devido ao facto dela ter evoluído com poucos sintomas, facto que na nossa amostra é conferido, uma vez que a idade mínima do aparecimento dos primeiros sintomas foi de 15 e a máxima de $\mathbf{5 0}$ anos de idade.

O tempo médio que demorou a ser estabelecido o diagnóstico foi de 6,88 anos. É importante referir que existem pacientes cujo diagnóstico foi confirmado em menos de um ano, no entanto num paciente o tempo que demorou a ser estabelecido o diagnóstico foi de 39 anos, o que, como afirmam pesquisadores da área, "(...) atrasos de 10 anos são o exemplo padrão de crime clínico"24,21,8. Considerando o facto de se tratar de uma doença com um comprometimento axial rápido e progressivo, estes 6,88 anos (média) podem ser considerados elevados e podem ter consequências nefastas, uma vez que a evolução poderia ter sido contrariada em fases precoces da doença, e que, devido ao aumento do tempo de confirmação, a doença foi deixada evoluir.

No presente estudo, foi possivel verificar que todos os doentes com aproximadamente onze anos de evolução se apresentam profissionalmente activos e com profissões diversas, o que de acordo com estudos, é gratificante para os doen-

\begin{tabular}{|c|c|c|c|}
\hline Componentes & Idade & TED & ID \\
\hline Função Física (FF] & $-0,582 * *(p=0,009)$ & $-0,531 *(p=0,028)$ & $-0,655^{* *}[p=0,003)$ \\
\hline Desempenho Físico (DF) & ns & $-0,570 *(p=0,017)$ & $-0,544 *(p=0,002)$ \\
\hline Dor Corporal (DC) & $0,530 * *(p=0,020)$ & $0,658^{* *}[p=0,004)$ & $0,700 * *(p=0,001)$ \\
\hline Saúde Geral [SG] & ns & ns & ns \\
\hline Vitalidade (VT) & ns & $0,497 *(p=0,042)$ & $0,557 *(p=0,016)$ \\
\hline Função Social [FS] & ns & $0,529 *(p=0,029)$ & ns \\
\hline Desempenho emocional (DE) & ns & ns & ns \\
\hline Saúde Mental (SM] & ns & ns & ns \\
\hline
\end{tabular}

QUADRO7

Associações entre Idade, tempo de estabelecimento diagnóstico, idade do diagnóstico e as componentes do SF-36.

[*] Correlação significativa com nível de significância $(\alpha=0,05)$; ( * ] Correlação significativa com nível de significância $(\alpha=0,01$ ); TED: Tempo de estabelecimento do diagnóstico; ID: Idade do diagnóstico; ns: diferença não significativa; $\boldsymbol{p}$ : nível de significância; tes e que vai de encontro com um estudo realizado na Noruega ${ }^{6}$, sobre um universo de 100 pacientes com EA, que revelou que um pouco mais de metade se mantinha profissionalmente activa ao fim de 16 anos de evolução, sendo, por vezes necessárias algumas intervenções ergonómicas.

Relativamente ao estado civil, apurou-se que a maioria era casada, o que permite corroborar a ideia ${ }^{27}$, de que as pessoas com EA têm geralmente uma vida conjugal produtiva e gratificante, podendo casar e criar os filhos como qualquer outro cidadão. No entanto estudos realizados demonstraram que, por vezes, surgem problemas relacionados com o sexo, uma vez que o doente pode não desejar o contacto sexual em consequência das dores, da fadiga, da anemia e da falta de líbido.

\begin{tabular}{lccc} 
Componentes & EF & Não EF & $p$ \\
\hline Função Física (FF) & 65,00 & 33,89 & 0,003 \\
Desempenho Físico (DF) & 65,00 & 33,33 & 0,000 \\
Dor Corporal (DC) & 46,00 & 68,89 & 0,028 \\
Saúde Geral (SG) & 63,00 & 60,00 & ns \\
Vitalidade (VT) & 47,50 & 54,17 & 0,043 \\
Função Social (FS) & 48,75 & 51,39 & ns \\
Desempenho emocional (DE) & 84,17 & 50,00 & 0,001 \\
Saúde Mental (SM) & 61,00 & 47,78 & 0,000 \\
\hline & & & QUADRo8
\end{tabular}

Diferença de médias de acordo com o género.

EF: Pratica exercício físico; Não EF: Não pratica exercício físico; ns: diferença não significativa; $\boldsymbol{p}$ : nível de significância; 


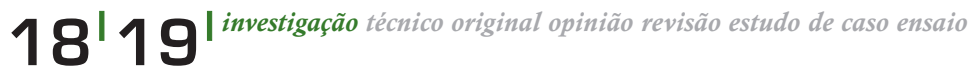

Relativamente à reabilitação, analisou-se que a maioria realiza reabilitação periodicamente e com o intuito de tratamento dos sintomas e de prevenir a evolução da doença, o que demonstra uma preocupação da parte dos pacientes em tentar reduzir os sintomas e minimizar a evolução progressiva que caracteriza a doença. Relativamente à modalidade terapêutica, a maioria realiza hidroterapia, o que vai de encontro a estudos realizados e que concluem tratar-se da modalidade terapêutica mais praticada e que demonstra contribuir para o aumento da funcionalidade ${ }^{1}$, diminuição da dor e melhoria da percepção global da saúde ${ }^{32}$ e possui um papel importante na reversão do quadro inicial da doença ${ }^{2}$.

Pesquisadores expressam que se alguém encontrar uma pessoa nova a sofrer à pouco tempo desta doença poucas coisas terá de retirar-lhe do seu projecto de vida, mas há uma a acrescentar-lhe e para sempre. Esse encargo acrescentado consiste em ter de desfazer todos os dias, a rigidez que a doença espondilítica tiver originado nas 24 horas anteriores ${ }^{24}$. Neste estudo conferiu-se que dez pacientes realizavam exercício físico e os restantes nove não cumpriam qualquer plano de exercício físico, o que demonstra que aproximadamente metade da amostra não realiza exercício físico, facto que é negativo tanto ao nível da prevenção ou retardamento da evolução, como da melhoria da funcionalidade e diminuição da anquilose.

Os motivos que conduzem os pacientes a praticarem o exercício físico, foram o de obter benefícios ao nível do estado da saúde, e com a finalidade de contrariar a evolução da doença. Os factores aconselha- mento médico e convívio também foram abordados como motivos para praticar exercício físico, o que, em estudos realizados, demonstram que a prática de exercício físico na EA apresenta benefícios físicos, conjuntamente com benefícios psicológicos, resultantes do facto dos pacientes compartilharem as suas próprias experiências entre $\mathbf{s i}^{10}$.

Aquando da verificação da relação entre idade e as dimensões da qualidade de vida, investigadores ${ }^{14}$, referem que com a idade as pontuações médias diminuem significativamente, o que vai de encontro aos resultados obtidos no presente estudo. Obtiveram-se relações entre a idade e a componente $F F$, em que o aumento da idade está associado a uma diminuição da capacidade de executar actividades físicas menores, como tomar banho ou vestir-se, actividades extenuantes, actividades intermédias, como levantar ou carregar as compras, subir escadas, inclinar-se, ajoelhar-se e baixar-se. As relações positivas entre a idade e a DC descobertas permitiram aferir que, com o passar dos anos e com a evolução da doença, a dor corporal aumenta, o que é importante, uma vez que adversa os resultados de um estudo realizado em Espanha ${ }^{3}$, que demonstrou que, com o passar dos anos, a dor corporal diminui em resultado da anquilose.

Pesquisas realizadas demonstraram que o aumento do tempo [anos] de confirmação do diagnóstico se relaciona com a qualidade de vida ${ }^{33}$. As correlações negativas situaram-se nas dimensões FF, DF e as positivas, ao nível da DC, em que o aumento do tempo de estabelecimento do diagnóstico se encontra positivamente associado com o aumento da dor corporal. As compo- nentes FS e VT também se relacionaram com o aumento do tempo de estabelecimento do diagnóstico. Quanto mais tempo demora a confirmação do diagnóstico, maiores são os níveis de FS, que representam a quantidade e qualidade das actividades sociais e o impacto dos problemas físicos e emocionais nestas actividades, e maiores os níveis de VT. Contudo, os níveis de dor também aumentam com o aumento do tempo de estabelecimento do diagnóstico.

A idade do diagnóstico analogamente se relacionou com as componentes da qualidade de vida. Representa que, quanto mais tarde é diagnosticada, maiores são as pontuações médias ao nível das componentes DC e VT; ao ser diagnosticada mais tarde contribui para que a doença seja deixada evoluir e, consequentemente, aumente a dor percepcionada, no entanto, as pessoas sentem mais energia e força para viver quando a doença é diagnosticada em idades mais avançadas. As relações de associação negativas encontradas, entre o aumento da idade do diagnóstico e as componentes FF e do DF, sugerem que o desempenho e a função física diminuem com o aumento da idade do diagnóstico ${ }^{9}$.

A prática de exercício físico reporta benefícios ao nivel de diversas componentes da qualidade de vida. No presente estudo, estes benefícios foram conferidos, pois encontraram-se diferenças nas pontuações médias das componentes entre os praticantes e os não praticantes. Provou-se que os doentes que praticavam exercício físico apresentavam valores médios superiores nas componentes função e desempenho físico e saúde mental, comparando com os pacientes que não 
praticavam. Estas diferenças podem ser compreendidas ${ }^{20}$, pelo facto do exercício físico, ao ser estruturado, planeado e repetitivo, ser benéfico ao nível da força muscular, amplitude articular, propriocepção, postura corporal e, consequentemente meIhorar performances funcionais.

Também se apuraram benefícios ao nível do desempenho emocional e da vitalidade que, podem ser aclarados devido ao facto do exercício físico possibilitar o aumento da motivação e eficácia, elevação da auto-estima, redução dos níveis de ansiedade e depressão ${ }^{7}$, todos eles factores fundamentais na intervenção terapêutica reabilitadora e no prognóstico dos doentes com EA. A componente DC similarmente apresentou benefícios com o exercício físico, isto porque este pode contribuir para o controlo da estimulação nociceptiva, quer pelo bloqueio a nível medular ["gate-control'], quer pela libertação de substâncias que limitam a actividade de neurotransmissores envolvidos nas mensagens dolorosas ao nível medular ${ }^{20}$.

De facto, após a verificação das diferenças nas médias das componentes, concluiu-se que estas são estatisticamente significativas. Tudo isto permite afirmar que o exercício físico regular assume-se como uma das opções mais válidas, cujos benefícios também têm sido evidenciados em inúmeros estudos $^{30,17,18,16,11}$, ao nível dos sintomas mais comuns desta patologia, nomeadamente na redução da dor e da rigidez articular, melhoria ou manutenção da amplitude articular, incremento da força muscular e melhoria da condição física, com repercussões positivas na funcionalidade e na percepção holística do estado de saúde.

\section{CONCLUSÃO}

Com as análises referentes aos dados obtidos, cujo principal objectivo foi verificar a relação entre a prática de exercício físico e a qualidade de vida em relação com a saúde em doentes com Espondilite Anquilosante, conclui-se que a qualidade de vida em relação com a saúde, apesar das diferenças não serem estatisticamente significativas, é diferente entre o género, sendo que o feminino apresenta valores inferiores ao masculino na maioria das componentes. As variáveis idade, idade do diagnóstico e tempo de estabelecimento do diagnóstico influenciam na percepção do estado de saúde dos doentes e o exercício físico aparece inegavelmente como uma modalidade terapêutica e promotor da qualidade de vida.

Motricidade Humana é o "corpo em acto", por isso os profissionais desta área do saber devem planear sessões de exercícios, criar classes de doentes onde se possam realizar exercícios terapêuticos com o intuito de impedir ou contrariar todo o processo evolutivo inerente à doença e, deste modo, influenciar na qualidade de vida destes doentes.

A Espondilite Anquilosante constitui uma das doenças com mais implicações psico-familiares e socioprofissionais, na medida em que influencia ao nível de absentismo laboral e das reformas precoces. Pensamos que é necessário transmitir aos doentes que, se realmente estiverem dispostos a trabalhar/praticar exercício físico, podem conseguir uma redução da dor e melhorias substanciais nos seus níveis de actividade e qualidade de vida.

\section{AGRADECIMENTOS}

Agradeço à Universidade Fernando Pessoa pelo apoio incondicional, à Prof. Doutora Dolores Monteagudo pela orientação e a todos os que possibilitaram a realização da investigação nomeadamente ao Prof. Doutor Pedro Lopes Ferreira, à professora Fernanda Faria e aos pacientes pelo contributo e disponibilidade em participar no estudo.

\section{CORRESPONDÊNCIA}

Rosa Maria Dantas Costa

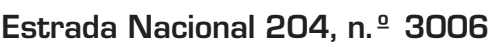

Porca - Facha

4990-600 Ponte de Lima

Email: rositafaxa@iol.pt 10989@ufp.pt

\section{REFERÊNCIAS}

1. Altan L, Bingol U, Aslan M, Yurtkuran $M$ (2006). The effect of balneotherapy on patients with Ankylosing Spondylitis. Scandinavian Journal of rheumatology, 35 (4), pp. 283-289. 2. Antunes R, Martelo D, Marques E (2005). Espondilite anquilosante: da doença inflamatória á reabilitação, Arquivos de Fisiatria, 12 (45, Jan./Fev./Mar.J, pp. 13-24.

3. Ariza-Ariza R, Hernandez-Cruz B, Navarro-Sarabia F (2003). Physical function and health-related quality of life of Spanish patients with Ankylosing Spondylitis, Arthritis and Rheumatism, 49 (4), pp. 483-487. 4. Bostan E, Borman P, Bodur H, Barca N (2003). Functional disability and quality of life in patients with Ankylosing Spondylitis, Rheumatology International, 23(3), pp. 121-126. 
5. Cardoso J (2000). Doenças metabólicas e exercício físico. In: Pinto $A$ [1 ${ }^{\mathrm{a}}$ ed.]. Saúde e exercício. Coimbra, Editora Quarteto, pp. 75-95.

6. Chorus S, Miedema S, Boonen A, Van der Linden S (2003). Quality of life and work in patients with rheumatoid arthritis and Ankylosing Spondylitis of working age, Annals of the rheumatic disease, 62(12), pp. 1178-1184.

7. Coutinho V (2000). 0 exercício físico e a clínica geral. In: Pinto $A$ (1a edição). Saúde e exercício físico. Coimbra. Editora quarteto, pp. 106-116.

8. Dagfinrud H, Mengshoel M, Hagen, B, Loge H, Kvien K [2004). Health States of patients with Ankylosing Spondylitis: a comparison with the general population, Annals of the rheumatic disease, 63(12), pp. 1605-1610.

9. Davis C, Van der Heijde D, Dougados M, Woolley M (2005). Reductions in health quality of life in patients with Ankylosing Spondylitis and improvements with etanercept therapy, Arthritis and rheumatism, 53(4), pp. 494-501.

10. Dziedzic K (2000). Espondilitis Anquilosante. In: David C, Lloyd J Rehabilitación Reumatológica. Madrid. Editora Harcourt, pp. 119-139. 11. Fernandez-de-Las-Penas C, Alonso-Blanco C, Morales-Cabezas M, Miangolarra-Page JC (2005). Two exercise interventions for the management of patients with Ankylosing Spondylitis: a randomized controlled trial, American journal of physician medicine and rehabilitation, 84(6), pp. 407-419.

12. Ferreira $P$ (2000). Criação da versão portuguesa do SF-36 - Parte I - Adaptação cultural e linguística, Acta Médica Portuguesa, 13, pp. 55-66.

13. Ferreira $P$ (2000). Criação da versão portuguesa do SF-36 - Parte II - Testes de validação, Acta Médica Portuguesa, 13, pp. 119-127.
14. Ferreira P, Santana P (2003). Percepção de estado de saúde e de qualidade de vida da população activa: contributo para a definição de normas portuguesas, Revista portuguesa de saúde pública, 2 [2, Jul-dez), pp. 15-30.

15. Haibel H, Niewerth M, Brandt J, Rudwaletit M, Listing J, Sieper J, Braun J (2004). Measurement of quality of life in patients with active Ankylosing Spondylitis being treated with infliximab - a comparison of SF-36 and SF-12, Zeitschript fur Rheumatologie, 63[5], pp. 393-401. 16. Ince G, Sarpel T, Durgun B, Erdogan $S$ (2006). Effects of a multimodal exercise program for people with Ankylosing Spondylitis, Physical Therapy, 86(7),pp.924-935. 17. Lim J, Lee S, Lim S (2005). Exercise, pain, perceived family support, and quality of life in Korean patients with Ankylosing Spondylitis, Psychological reports, 96(1), pp. 3-8.

18. Lim J, Moon I, Lee S (2005). Effects of home-based daily exercise therapy on joint mobility, daily activity, pain, and depression in patients with Ankylosing Spondylitis, Rheumatology International, 25 (3), pp. 225-229.

19. Mediavilla M, Ferreira J (1998). Espondilite Anquilosante, Pathos [Formação médica contínua], XIV [3], pp. 33-51.

20. Pinheiro J (2000). O exercício físico como reabilitação. In: Pinto A [1 a edição]. Saúde e exercício físico. Coimbra. Editora quarteto, pp.57-63. 21. Queiroz M (1998). Reumatologia Clínica. Lisboa, Edições Lidel. 22. Queiroz M (2002). Espondilartrite Anquilosante. In: Queiroz M. Reumatologia - Clínica e terapêutica das doenças reumáticas I. Lisboa. Edições Lidel, pp. 230-239. 23. Ribeiro J (1997). A promoção da saúde e da qualidade de vida em pessoas com doenças crónicas. In: Ribeiro J. Actas - Sociedade Portuguesa de Psicologia da Saúde. Braga. Editado pelo Instituto Su- perior de Psicologia Aplicada, pp. 253-272.

24. Rocha F (2002). Manual da Espondilite Anquilosante. Lisboa, Secretariado Nacional para a reabilitação e integração das pessoas com deficiência.

25. Rocha F (2002). Espondilite Anquilosante - os factos. Alcabideche, direcção da ANEA.

26. Sérgio M (2005). Motricidade Humana - qual o futuro?, Motricidade, 1(4), pp. 271-283.

27. Sieper J, Braun J [2006). Biologic Therapies in Spondyloarthrities - The Current State. In: Royen B, Dijkmans B, (1 a edição). Ankylosing Spondylitis: Diagnosis and Management. New York, Editora Taylor \& Francis, p. 170.

28. Silva J [2004). Reumatologia Prática. Coimbra, Edições Diagnósteo. 29. Skare T (1999). Espondiloartropatias seronegativas - conceitos gerais. In: Skare T. Reumatologia Princípios e prática. Rio de Janeiro. Editora Guanabara Koogan, p. 173. 30. Sweeney S, Taylor G, Calin A (2002). The effect of home based exercise intervention package on outcome in Ankylosing Spondylitis: a randomized controlled trial. Journal of Rheumatology, 29 (4), pp. 763-766.

31. Timms A, Wordsworth B, Brown M (2006). Epidemiology, Pathogenesis, and Genetics of Ankylosing Spondylitis. In: Royen B, Dijkmans B (1 ${ }^{\text {a }}$ edição). Ankylosing Spondylitis: Diagnosis and Management. New York, Editora Taylor \& Francis, p. 23.

32. Van Tubergen A, Coenen J, Landewe R, Spoorenberg A, Chorus A, Boonen A, Van Der Linden S, Van Der Heidje D (2002). Assessment of fatigue in patients with Ankylosing spondylitis: a psychometric analysis, Arthritis and rheumatism, 47(1), pp. 8-16.

33. Ward M (1998). Quality of life in patients with Ankylosing Spondylitis, Rheumatology disease clinical North American,24(4), pp.815-827. 